\title{
First record of Tomato chlorotic spot virus in the USA
}

\author{
Aurora Londoño ${ }^{1}$, Heather Capobianco ${ }^{1}$, Shouan Zhang $^{2}$ \& Jane E. Polston ${ }^{1}$ \\ ${ }^{1}$ Dept. of Plant Pathology, University of Florida, Gainesville, FL 32611 USA; ${ }^{2}$ Dept. of Plant Pathology, Tropical Res. and \\ Educ. Ctr., University of Florida, Homestead, FL 33031 USA
}

Author for correspondence: Jane E. Polston, e-mail: jep@ufl.edu

\begin{abstract}
A tospovirus was identified in tomato plants from two counties in Florida by reverse transcription-PCR and sequencing of portions of the S, M and L genomic segments. The predicted amino-acid sequences of the N protein of PCR products from four plant samples were $>96 \%$ identical to those of TCSV. Partial nucleic acid sequences of the L and M RNA were $>97 \%$ identical to those reported for TCSV isolates. Extracts from field samples infected test plants and produced symptoms similar to those reported for TCSV. This is the first report of an isolate of TCSV in Florida and in the USA.
\end{abstract}

Key words: Bunyaviridae, Solanum lycopersicum, South Florida, Tospovirus.

Viruses in the genus Tospovirus, family Bunyaviridae are plant pathogens transmitted by thrips (Thysanoptera: Thripidae), with a tri-segmented ambisense RNA genome and the ability to replicate in thrips as well as plants. The diversity of the genus comprises 11 recognized species with a variable host range and geographic distribution, with an additional 15 proposed species (de Oliveira et al., 2012; Hassani-Mehraban et al., 2010; King et al., 2012). Species classification is based on serology, host range and nucleotide and predicted amino acid sequence of the $\mathrm{N}$ protein, which is located on the S RNA segment. Based in these criteria, tospoviruses have also been differentiated into American and Asiatic clades (de Oliveira et al., 2012; Hassani-Mehraban et al., 2010).

There are five tospoviruses reported from the USA: Groundnut ring spot virus (GRSV), Impatiens necrotic spot virus (INSV), Iris yellow spot virus (IYSV), Soybean vein necrosis associated virus (SVNaV), and Tomato spotted wilt virus (TSWV) (Pappu et al., 2009; Webster et al., 2010; Zhou et al., 2011). The first three viruses belong to the American clade whereas IYSV belongs in the Asiatic clade. None of these viruses are native to North America and were likely introduced (Pappu et al., 2009). An isolate of GRSV, GRSV- $\mathrm{L}_{\mathrm{G}} \mathrm{M}_{\mathrm{T}} \mathrm{S}_{\mathrm{G}}$, is a reassortant between GRSV and Tomato chlorotic spot virus (TCSV), and was reported from tomato in Florida in 2010 (Webster et al., 2011). This isolate is composed of the L and S RNA segments from GRSV but an M RNA more closely-related to that of TCSV than GRSV. GRSV- $\mathrm{L}_{\mathrm{G}} \mathrm{M}_{\mathrm{T}} \mathrm{S}_{\mathrm{G}}$ was proposed to have originated in an area where both TCSV and GRSV occur since neither virus had been reported from the USA at the time of the report (Webster et al., 2011).

During spring 2012, symptoms of systemic necrosis and leaf distortion were observed in tomato plants in fields in the Florida counties of Miami-Dade and Lee (Figure 1). Fifteen and 12 plants were collected from each county and tested for the presence of tospoviruses. Eight and six plants from Miami-Dade and Lee Co., respectively, gave positive reactions in an enzyme-linked immunosorbent assay (ELISA) designed to detect GRSV and TCSV (SRA6100, AgDia, Inc. Elkhart, IN). To confirm the identity of the virus, the samples were assayed by reverse transcription-PCR and sap extract from selected samples were mechanically inoculated to test plants.

RNA was extracted using a Tri-Reagent procedure (Ambion Inc. Grand Island, NY) and two step reverse transcription-PCR was performed to amplify regions of the three RNA segments using one primer pair for each segment. For amplification of a region of the S RNA, a pair of primers, J13/UH1 (Cortez et al., 2001), was used which amplifies the N ORF and the intergenic region of many tospoviruses and generates different sized amplicons due to variations in the length of the intergenic region among tospovirus species. Amplification of a region of the M RNA was performed with the primer pair NS1/CLA1 (Eiras et al., 2001). Amplification of a region of the L RNA was performed with degenerate primer gL4510c (Cortez et al., 2001) and a TCSV sequence specific primer, JAP891 (5'ACTGGGTCCACTTTACTCACTT3'), which was designed for this study using an L RNA sequence of an isolate of TCSV (GenBank Acc. No. HQ700667) (Bertran et al., 2011). Samples were also tested with a pair of primers designed to specifically amplify a region of the TCSV S RNA (JAP885/JAP886, 5'CTCGGTTTTCTGCTTTTC3'/ 5'CGGACAGGCTGGAGAAATCG3'-vc) and two sets of primers specific for the GRSV S RNA, JAP887/JAP888 (5 'CGTATCTGAGGATGTTGAGT3'/5'GCTAACTCCTTG TTCTTTTG3'-vc) and GRSV-N-v/GRSV-N-vc [12]. 

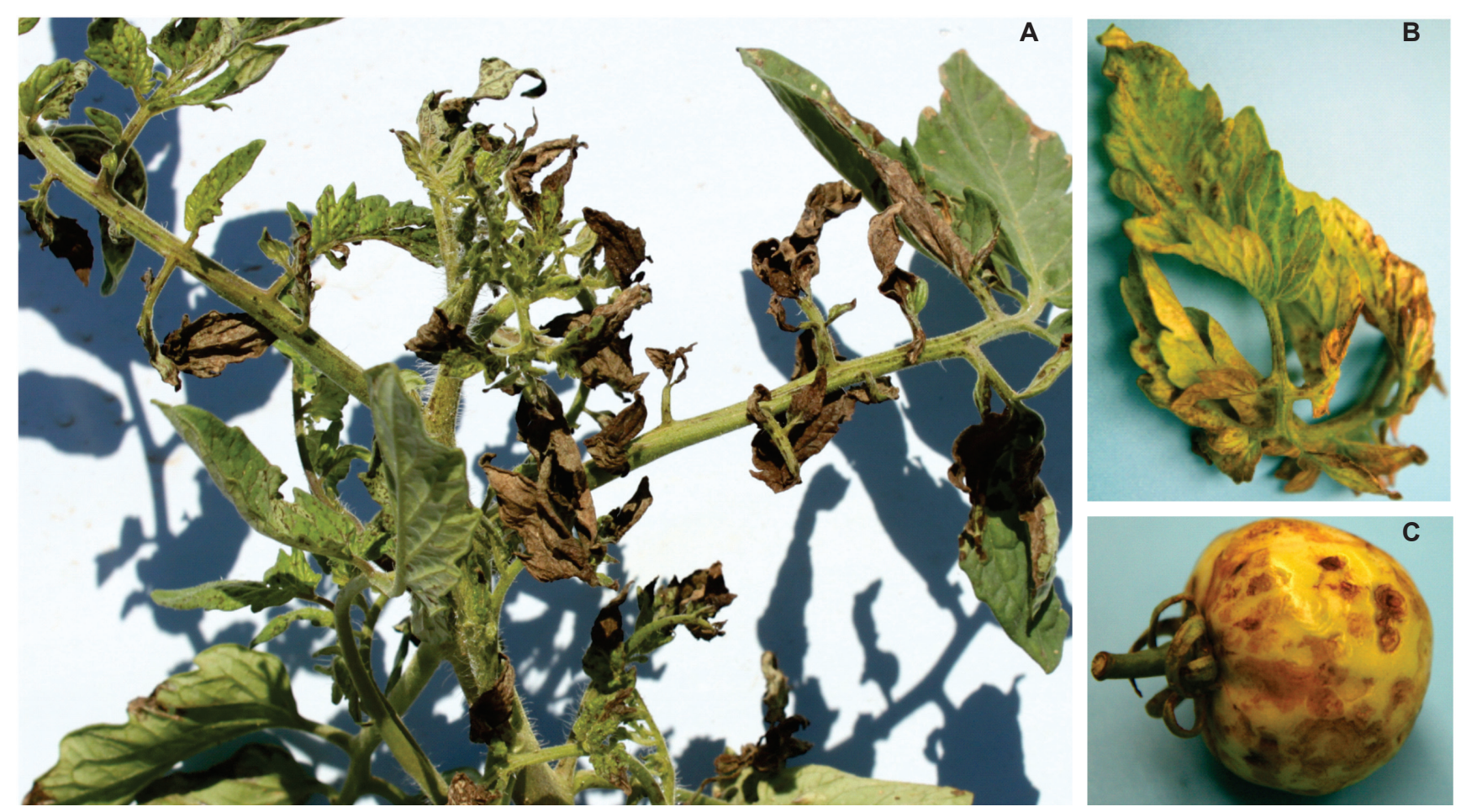

FIGURE 1 - Symptoms of Tomato chlorotic spot virus in tomato plants from Miami-Dade and Lee Counties in Florida. A. Tomato plant (two weeks after transplant) in Miami-Dade Co. showing symptoms of necrosis and deformation, similar plants were dead within a week after this image was taken; B. Tomato leaf from an infected mature plant from Lee Co. showing necrotic spots and deformation; C. Fruit from an infected mature plant from Lee Co. showing necrotic spots, necrotic ringspots and deformation.

Six samples from Miami-Dade Co. and seven samples from Lee Co. yielded the expected size amplicons of 1408-1455 (S RNA), 878 (M RNA) and 1532 (L RNA) bp for primer pairs J13/UH1, NS1/CLA1, and gL4510c/ JAP891, respectively. Primer pair J13/UH1 generated a single size amplicon from all 13 samples, suggesting that no other tospovirus was present in these samples. No sample amplified with primer pair JAP886/JAP887 or GRSV-N-V/ GRSV-N-vc suggesting that GRSV was not present in any of these samples. All the samples that gave the expected size amplicon using primer pair J13/UH1 also amplified the expected size amplicon of $295 \mathrm{bp}$ using the TCSV specific primer pair, JAP884/JAP885. Two samples from each county were selected and amplicons of the three segments from each of the four samples were cloned into pGEMT-Easy vector (Promega Corp. Madison WI), sequenced, and the sequences compared to those in GenBank through Blastn and Blastp. Sequences generated were deposited in the GenBank database as accession numbers JX244195 through JX244206.

Sequences obtained using the S RNA primer pair, J13/UH1, were 96-99\% identical with each other and were most closely related to variably sized sequences of the S RNA of TCSV. Sequences from Florida had nucleic acid sequence identities (NSI) of $97 \%$ with the $\mathrm{N}$ gene of TCSV-[BR-03] (GenBank Acc. No. S54325), which is the type isolate of TCSV (de Avila et al., 1993), and 98\% with a shorter segment (775 nt) of another isolate of TCSV from Brazil (GenBank Acc. No. AF282982) (Table 1). In contrast, the NSI of the isolates in this study were only $84 \%$ identical with the sequence of the $\mathrm{N}$ gene of a GRSV isolate from South Africa (GRSV-[95/0137], GenBank Acc. No. AF487517), 83\% with a sequence from Brazil (GenBank Acc. No. AF251271), and 80\% with GRSV- $\left[\mathrm{L}_{\mathrm{G}} \mathrm{M}_{\mathrm{T}} \mathrm{S}_{\mathrm{G}}\right]$ from Florida (Table 1). The NSI with other tospoviruses, including the recently proposed species Pepper necrotic spot virus (PNSV) (Torres et al., 2012), TWSV and INSV was $76 \%$ or lower.

The predicted amino acid sequences of the $\mathrm{N}$ protein of the sequences obtained in this study were almost identical to each other (Table 2). The percent of amino acid sequence identity (ASI) of these sequences with the respective protein sequences of TCSV ranged from 96 to $98 \%$, in contrast to those of GRSV and TSWV isolates which ranged from 87 to $88 \%$ and 80 to $81 \%$, respectively. Using the current criteria for taxonomy of the genus Tospovirus, which establishes $90 \%$ ASI in the N protein as the delimiting value for species demarcation (King et al., 2012), the virus identified in the samples would be considered isolates of TCSV.

Sequences obtained with primers for the M RNA had a NSI of $99 \%$ with each other. These sequences had an NSI of $99 \%$ with the equivalent region of GRSV- $\left[\mathrm{L}_{\mathrm{G}} \mathrm{M}_{\mathrm{T}} \mathrm{S}_{\mathrm{G}}\right]$ (GenBank Acc. No. HQ644141), followed by a NSI of 98\% with two TCSV sequences reported from Brazil (GenBank 
First record of Tomato chlorotic spot virus in the USA

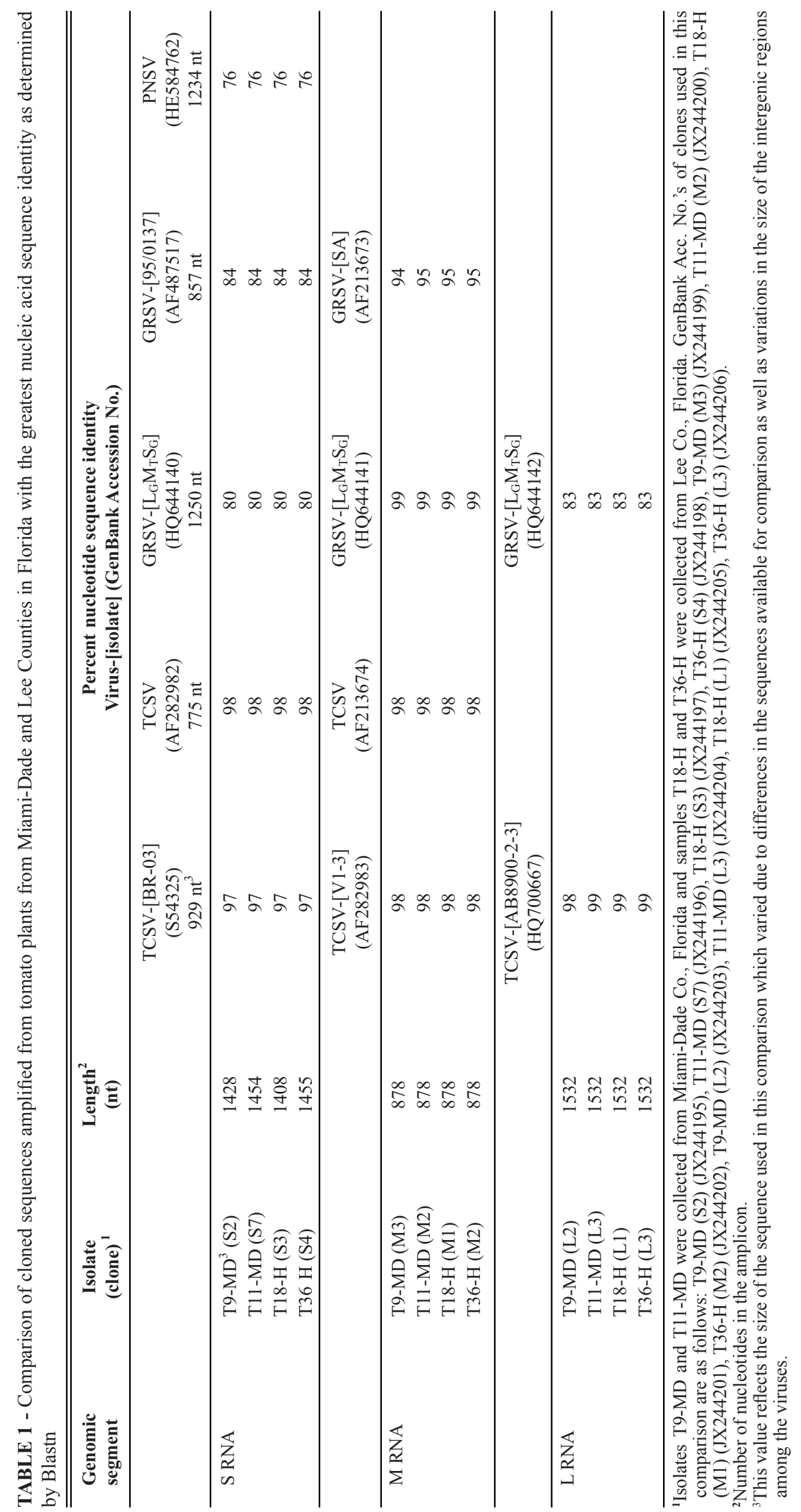


A. Londoño et al.

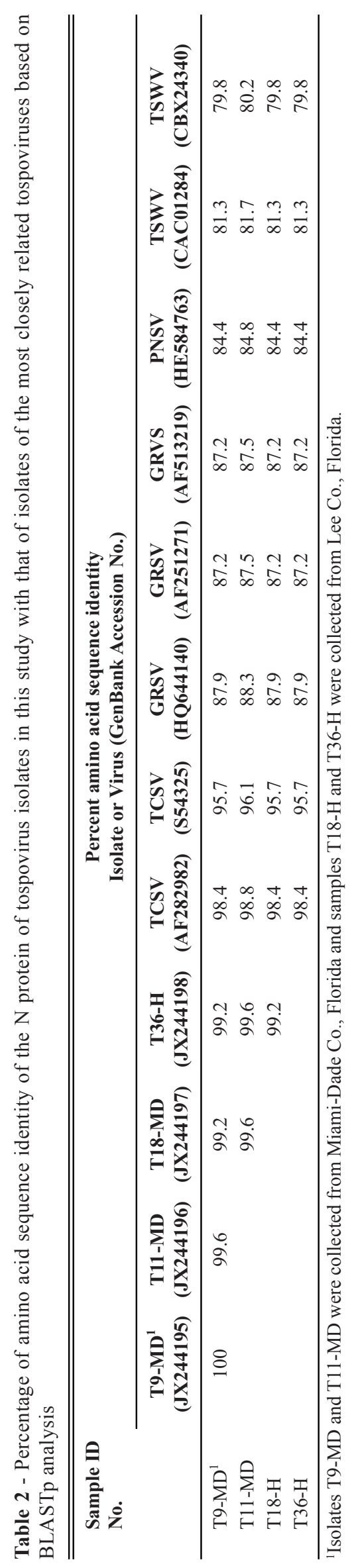


Acc. No. AF282983 and AF213674) (Table 1). The next most similar were isolates of GRSV reported from South Africa (GRSV-[SA] GenBank Acc. No. AF213673) and Brazil (GenBank Acc. No. AF213673, AF513220) with an NSI of $94-95 \%$ (Table 1). The translated sequence, 255 aa of the NSm protein had 100\% ASI with the equivalent sequence of GRSV-[ $\left.\mathrm{L}_{\mathrm{G}} \mathrm{M}_{\mathrm{T}} \mathrm{S}_{\mathrm{G}}\right], 99 \%$ ASI with the two TCSV isolates, and $98 \%$ and $96 \%$ ASI with GRSV isolates from South Africa and Brazil, respectively.

Amplicon sequences of the L RNA (primers gL4510c/JAP891) had NSIs of $99 \%$ to each other and 98 $99 \%$ with a portion of the coding sequence for the RNAdependent RNA polymerase (RdRp) of TCSV (GenBank Acc. No. HQ700667) [1] (Table 1). These sequences had only $83 \%$ NSI with the equivalent region of the L RNA from GRSV- $\left[\mathrm{L}_{\mathrm{G}} \mathrm{M}_{\mathrm{T}} \mathrm{S}_{\mathrm{G}}\right]$ (GenBank Acc. No. HQ644142) (Table 1). The NSI of the amplicon sequences had lower similitudes with the L RNA of other tospoviruses; $79-80 \%$ NSI with isolates of TSWV (GenBank Acc. No. HM581934, NC 002052,AB190813) and 76\% NSI with INSV (GenBank Acc. No. X93218) (data not shown). A predicted amino acid sequence of $510 \mathrm{nt}$, which corresponds to a portion of the Bunyaviridae RdRp domain, had 99\%, 96\%, 90-91\% and $80 \%$ of ASI with those of TCSV, GRSV- $\left[\mathrm{L}_{\mathrm{G}} \mathrm{M}_{\mathrm{T}} \mathrm{S}_{\mathrm{G}}\right]$, TSWV, and INSV (GenBank Acc. No. HQ700667, HQ644142, HM581934, X93218, respectively). These values indicate that the sequences obtained from field samples using $\mathrm{L}$ RNA primers gL4510c/JAP891are more similar to isolates of TCSV than to any other tospovirus including GRSV$\left[\mathrm{L}_{\mathrm{G}} \mathrm{M}_{\mathrm{T}} \mathrm{S}_{\mathrm{G}}\right]$.

To obtain biological information on the virus isolates, two samples from Miami-Dade Co. which tested positive for TCSV by ELISA and reverse transcription-PCR using primer pairs J13/UH1, NS1/CLA1, gL4510c/JAP891 and JAP884/JAP885 were mechanically inoculated to pepper (Capsicum annuum L. 'California Wonder') using a buffer composed of $0.1 \mathrm{M}$ sodium/potassium phosphate and $0.01 \mathrm{M}$ sodium sulfite, $\mathrm{pH}$ 7.4. Large chlorotic ring spots (which later developed into necrotic ring spots) appeared on the inoculated leaves eight days post inoculation. Systemic symptoms of leaf distortion and curling and veinal chlorosis appeared within nine days. A chlorotic lesion from pepper was collected and inoculated to pepper seedlings, Datura stramonium L., and Nicotiana glutinosa L. Pepper plants inoculated with the local lesion produced symptoms identical to the originally inoculated plant, while $D$. stramonium and $N$. glutinosa plants exhibited necrotic lesions on the inoculated leaf four days after inoculation. Seven days after inoculation, systemic symptoms of necrotic spots, leaf distortion and leaf curling appeared on plants of D. stramonium. All inoculated symptomatic plants tested positive by reverse transcription-PCR with primers JAP884/ JAP885 and the GRSV/TCSV ELISA. Additionally, direct sequencing of reverse transcription-PCR products for $\mathrm{S}, \mathrm{M}$, and L RNA (primer pairs gL4510c/JAP891, NS1/CLA1 and J13/UH1, respectively) from pepper and D. stramonium plants yielded sequences $100 \%$ identical to the clones from the tomato field samples.

TCSV was not detected in every field plant with necrotic symptoms. The lack of detection of a tospovirus in every necrotic sample was most likely due to the poor quality of the plant tissue. We have found that nucleic acid extractions of necrotic tissue contain plant compounds associated with the necrosis (ie tanins etc.) which can interfere with reverse transcription PCR.

The symptoms observed in tomato plants from the field, combined with ELISA results and sequence analyses of twelve amplicons obtained from four field samples using six primer pairs are consistent with the presence of TCSV in two locations in Florida. The symptoms in tomato in the field and in the test plants (C. annuum, D. stramonium, $N$. glutinosa) are consistent with those reported for a TCSV isolate from Brazil (de Avila et al., 1993). The sequences of all amplicons had greater than $97 \%$ NSI with sequences of TCSV isolates. The predicted amino acid sequence of the $\mathrm{N}$ protein of the isolates in this study had greater than 95\% ASI with TCSV isolates. While the M RNA of TCSV has been reported previously as part of the genome of the reassortant virus, GRSV-[ $\mathrm{L}_{\mathrm{G}} \mathrm{M}_{\mathrm{T}} \mathrm{S}_{\mathrm{G}}$ ] (Webster et al., 2011), no evidence for the presence of any segment of GRSV was found in these samples using reverse transcription-PCR and three primer pairs. These data support the conclusion that an isolate of TCSV is present in Florida and in the USA.

It is not clear how or when TCSV was introduced into Florida. Several TCSV vector species have existed in Florida for some time (Frantz \& Fasulo, 1997). However, the dark form of Frankliniella schultzei, demonstrated in one study to be the most efficient vector of TCSV, has only recently become a pest in vegetable crops in South Florida (Wijkamp et al., 1995; Kakkar et al., 2012). It is possible that TCSV is a recent introduction possibly on an unknown ornamental host. Miami-Dade Co. produces a very wide range of ornamental crops. It is also possible that TCSV has been in Florida for some time, and only recently become apparent due to the increase in populations of $F$. schultzei.

\section{ACKNOWLEDGEMENTS}

The authors would like to acknowledge the support of A. Londoño through an Innovation Fund Grant provided by the University of Florida, Office of the Dean for Research and support from the USDA-T/STAR program.

\section{REFERENCES}

Bertran AG, Oliveira AS, Nagata T, Resende RO (2011) Molecular characterization of the RNA-dependent RNA polymerase from Groundnut ring spot virus (genus Tospovirus, family Bunyaviridae). Archives of Virology 156:1425-1429.

Chu FH, Chao CH, Chung MH, Chen CC, Yeh SD (2001) Completion of the genome sequence of Watermelon silver 
mottle virus and utilization of degenerate primers for detecting tospoviruses in five serogroups. Phytopathology 91:361-368.

Cortez I, Saaijer J, Wongjkaew KS, Pereira AM, Goldbach R, Peters D, Kormelink R (2001) Identification and characterization of a novel tospovirus species using a new RT-PCR approach. Archives of Virology 146:265-278.

de Avila AC, de Haan P, Kormelink R, Resende Rde O, Goldbach RW, Peters D (1993) Classification of tospoviruses based on phylogeny of nucleoprotein gene sequences. Journal of General Virology 74:153-159.

de Oliveira AS, Melo FL, Inoue-Nagata AK, Nagata T, Kitajima EW, Resende RO (2012) Characterization of Bean becrotic mosaic virus: A member of a novel evolutionary lineage within the genus Tospovirus. PLoS ONE 7: e38634.

Eiras M, Resende RO, Missiaggia AA, de Avila AC (2001) RTPCR and Dot Blot hybridization for an universal detection of tospoviruses. Fitopatologia Brasileira 26:170-175.

Frantz G, Fasulo TR (1997) THRIPS: A knowledgebase of Vegetable Thrips: Glades Crop Care. Available at: http://www. gladescropcare.com/GCC_software.html

Hassani-Mehraban A, Botermans M, Verhoeven JT, Meekes E, Saaijer J, Peters D, Goldbach R, Kormelink R (2010) A distinct tospovirus causing necrotic streak on Alstroemeria sp. in Colombia. Archives of Virology 155:423-428.

Kakkar G, Seal DR, Kumar, V (2012) Assessing abundance and distribution of an invasive thrips Frankiliniella schulitzei
(Thysanoptera: Thripidae) in south Florida. Bulletin of Entomological Research 102:249-259.

King AMQ, Adams MJ, Carstens EB, Lefkowitz EJ (2012) Ninth Report of the International Committee on Taxonomy of Viruses. San Diego CA. Elsevier.

Pappu HR, Jones RAC, Jain RK (2009) Global status of tospovirus epidemics in diverse cropping systems: Successes achieved and challenges ahead. Virus Research 141:219-236.

Torres R, Larenas J, Fribourg C, Romero J (2012) Pepper necrotic spot virus, a new tospovirus infecting solanaceous crops in Peru. Archives of Virology 157:609-615.

Wijkamp I, Almarza N, Goldbach R, Peters, D (1995) Distinct levels of specificity in thrips transmission of tospoviruses. Phytopathology 85:1069-1074.

Webster CG, Perry K, Lu X, Horsman L, Frantz G, Mellinger C, Adkins ST (2010) First report of Groundnut ring spot virus infecting tomato in south Florida. Plant Health Progress doi:10.1094/PHP2010-0707-01-BR.

Webster CG, Reitz SR, Perry KL, Adkins S (2011) A natural M RNA reassortant arising from two species of plant- and insectinfecting bunyaviruses and comparison of its sequence and biological properties to parental species. Virology 413:216-225.

Zhou J, Kantartzi, SK, Wen, R-H, Newman M, Hajimorad MR, Rupe JC, Tzanetakis, IE. (2011) Molecular characterization of a new tospovirus infecting soybean. Virus Genes 43:289-295.

TPP-2012-0052 - Received 4 July 2012 - Accepted 31 July 2012

Section Editor: F. Murilo Zerbini 